\title{
ON IRREDUCIBLE DIVISORS OF ITERATED POLYNOMIALS
}

\author{
DOMINGO GÓMEZ-PÉREZ, ALINA OSTAFE, AND IGOR E. SHPARLINSKI
}

\begin{abstract}
D. Gomez, A. Ostafe, A. P. Nicolás and D. Sadornil have recently shown that for almost all polynomials $f \in \mathbb{F}_{q}[X]$ over the finite field of $q$ elements, their iterations eventually become reducible polynomials over $\mathbb{F}_{q}$. Here we combine their method together with some new ideas to derive series of finer results about the arithmetic structure of iterations of $f$. In particular, we prove that the $n$th iteration of $f$ has a square-free divisor of degree of order at least $n^{1+o(1)}$ as $n \rightarrow \infty$ (uniformly over $q$ ).
\end{abstract}

\section{INTRODUCTION}

For a field $\mathbb{K}$ and a polynomial $f \in \mathbb{K}[X]$ we define the sequence:

$$
f^{(0)}(X)=X, \quad f^{(n)}(X)=f\left(f^{(n-1)}(X)\right), \quad n=1,2, \ldots .
$$

The polynomial $f^{(n)}$ is called the $n$th iterate of the polynomial $f$.

Following $[1,2,10,11,14]$, we say that a polynomial $f \in \mathbb{K}[X]$ is stable if all iterates are irreducible over $\mathbb{K}$.

Gomez and Nicolás [7], developing some ideas from [15], have proved that there are $O\left(q^{5 / 2}(\log q)^{1 / 2}\right)$ stable quadratic polynomials over a finite field of $q$ elements $\mathbb{F}_{q}$ for an odd prime power $q$, where the implied constant is absolute. We also note that in [8] an upper bound is given on the number of stable polynomials of degree $d \geqslant 2$ over $\mathbb{F}_{q}$.

Here, we continue to study the arithmetic properties of iterated polynomials and obtain several new results about their multiplicative structure.

First, we combine the method of Gomez and Nicolás [7] with some new ideas to show that for almost all quadratic polynomials $f \in \mathbb{F}_{q}[X]$ the number $r_{n}(f)$ of irreducible divisors of the $n$th iterate $f^{(n)}$ grows at least linearly with $n$ if $n$ is of order up to $\log q$. Using this result, we prove that the largest degree of the irreducible divisors of $f^{(n)}$ grows with $n$ as well. Our tools to prove this are resultants of iterated polynomials, the Stickelberger's Theorem [18] and estimates of certain character sums. 
Beyond this threshold, we use a different technique, related to Mason's proof of the $A B C$-conjecture in its polynomial version, see $[12,17]$ and also a lower bound on the largest degree $D_{n}(f)$ of the irreducible divisors of $f^{(n)}$.

Note that our lower bounds on $D_{n}(f)$ are reminiscent of lower bounds on the largest prime divisor of nonlinear recursive sequences over the integers, see $[4,10,16]$.

The approaches and some results used to derive lower bounds on $r_{n}(f)$ and $D_{n}(f)$ are readily combined to obtain the lower bound $n^{1+o(1)}$ as $n \rightarrow \infty$ (uniformly over $q$ ) on the largest degree of square-free divisors of $f^{(n)}$.

The outline of the paper is the following. In Section 2 we give the notation used throughout the paper as well as collecting some basic properties needed in the proofs of the main results. In Section 3, we collect all results about discriminants and then, in Section 4, we provide bounds on character sums related with discriminants of iterated polynomials. In Section 5 we recall the result of Mason [12]. These preliminary results are used in the follow-up sections. More precisely, Section 6 contains an estimate of the number of irreducible factors in the iterations of polynomials. In Section 7 we show that, if $f \neq f_{d} X^{d}$, then there is always an irreducible factor of large degree for high order iterates of the polynomial $f$. Finally, in Section 8 we combine both approaches and also use some of the previous results to derive some nontrivial information about the arithmetic structure of $f^{(n)}$ that applies to any $n$.

\section{NotATiON}

Let $p$ be an odd prime number and $q=p^{s}$ for some positive integer $s$. We denote by $\mathbb{F}_{q}$ the finite field of $q$ elements and $\chi$ denotes the quadratic character of $\mathbb{F}_{q}$.

We use $\mathbb{F}_{q}[X]$ to denote the ring of polynomials with coefficients in $\mathbb{F}_{q}$. Polynomials in this ring are denoted by the letters $f, g$ and $h$. We usually use $f_{0}, \ldots, f_{d}$ to represent the coefficients of a polynomial $f \in \mathbb{F}_{q}[X]$, that is,

$$
f=f_{d} X^{d}+\ldots+f_{1} X+f_{0},
$$

where $f_{d} \neq 0$ is the leading coefficient of $f$. As usual, $f^{\prime}$ denotes the formal derivative of $f \in \mathbb{F}_{q}[X]$.

Throughout the paper the implied constants in symbols ' $O$ ' and ' $\gg$ ' may occasionally, where obvious, depend on the small positive parameter $\varepsilon$ and are absolute otherwise (we recall that $A=O(B)$ and 
$B \gg A$ is equivalent to $|A| \leqslant c B$ for some constant $c$ ). Also, we write $F(n)=o(G(n))$ as $n \rightarrow \infty$, which means that

$$
\lim _{n \rightarrow \infty} \frac{F(n)}{G(n)} \rightarrow 0
$$

\section{Discriminants and Iterations of Polynomials}

We use the following well known properties of discriminants Disc $(f)$ and resultants $\operatorname{Res}(f, g)$ of polynomials $f, g \in \mathbb{K}[X]$, see $[6,19]$, that hold over any field $\mathbb{K}$.

Lemma 1. Let $f, g \in \mathbb{K}[X]$ be polynomials of degrees $d \geqslant 1$ and $e \geqslant 1$, respectively, with leading coefficients $f_{d}$ and $g_{e}$, and let $h \in \mathbb{K}[X]$. Suppose that the derivative $f^{\prime}$ is a polynomial of degree $k \leqslant d-1$ and denote by $\beta_{1}, \ldots, \beta_{e}$ the roots of $g$ in an extension field. Then we have:

(i) $\operatorname{Disc}(f)=(-1)^{\frac{d(d-1)}{2}} f_{d}^{d-k-2} \operatorname{Res}\left(f, f^{\prime}\right)$;

(ii) $\operatorname{Res}(f, g)=(-1)^{d e} g_{e}^{d} \prod_{i=1}^{e} f\left(\beta_{i}\right)$;

(iii) $\operatorname{Res}(f g, h)=\operatorname{Res}(f, h) \operatorname{Res}(g, h)$.

From the definition of the resultant, it is clear that two polynomials $f$ and $g$ are co-prime if and only if $\operatorname{Res}(f, g) \neq 0$.

To study the discriminant of iterations of polynomials, it is necessary to have a close formula for the resultant of polynomials under compositions. In [13], the following chain rule for resultants is proved:

Lemma 2. Let $f, g$ be as in Lemma 1 and let $h \in \mathbb{K}[X]$ with $\operatorname{deg} h=\ell$ and leading coefficient $h_{\ell}$. Then

$$
\operatorname{Res}(f(h), g(h))=\left(h_{\ell}^{\text {de }} \operatorname{Res}(f, g)\right)^{\ell} .
$$

It is clear from Lemma 2 that $f$ and $g$ are co-prime if and only if for any nonconstant polynomial $h$ we have $\operatorname{Res}(f(h), g(h)) \neq 0$ (note that this is also a consequence of the Euclidean algorithm).

Also, Lemma 2 implies the following formula for the discriminant of polynomial iterates:

Lemma 3. Let $f \in \mathbb{F}_{q}[X]$ be a polynomial of degree $d \geqslant 2$ with leading coefficient $f_{d}$ and non-constant derivative $f^{\prime}$ of degree $k \leqslant d-1$. Suppose that $\gamma_{i}, i=1, \ldots, k$, are the roots of the derivative $f^{\prime}$. Then, for $n \geqslant 1$, we have

$$
\begin{array}{r}
\operatorname{Disc}\left(f^{(n)}\right)=(-1)^{d\left(\frac{d(d-1)}{2}+k\right)} f_{d}^{\frac{d^{n}-1}{d-1}\left((k-1) d^{n}+k \frac{d^{n}-d}{d-1}+2 d\right)}\left((k+1) f_{k+1}\right)^{d^{n}} \\
\operatorname{Disc}\left(f^{(n-1)}\right)^{d} \prod_{i=1}^{k} f^{(n)}\left(\gamma_{i}\right) .
\end{array}
$$


Proof. Simple calculations show that the leading coefficient of $f^{(n)}$ is

$$
f_{d}^{\frac{d^{n}-1}{d-1}} \text { and } \quad \operatorname{deg}\left(f^{(n)}\right)^{\prime}=k \frac{d^{n}-1}{d-1} \quad \text { for } n \geqslant 2 .
$$

Thus, applying Lemma 1(i) we derive

$$
\begin{aligned}
\operatorname{Disc}\left(f^{(n)}\right) & =(-1)^{\frac{d^{n}\left(d^{n}-1\right)}{2}} f_{d}^{\frac{d^{n}-1}{d-1}\left(d^{n}-k \frac{d^{n}-1}{d-1}-2\right)} \operatorname{Res}\left(f^{(n)},\left(f^{(n)}\right)^{\prime}\right) \\
& =(-1)^{\frac{d^{2}(d-1)}{2}} f_{d}^{\frac{d^{n}-1}{d-1}\left(d^{n}-k \frac{d^{n}-1}{d-1}-2\right)} \operatorname{Res}\left(f^{(n)},\left(f^{(n)}\right)^{\prime}\right) .
\end{aligned}
$$

Taking into account that $\left(f^{(n)}\right)^{\prime}=f^{\prime} \cdot\left(f^{(n-1)}\right)^{\prime}(f)$ and applying Lemmas 1(iii) and 2, we derive

$$
\begin{aligned}
& \operatorname{Res}\left(f^{(n)}, f^{(n) \prime}\right)=\operatorname{Res}\left(f^{(n)}, f^{\prime} \cdot\left(f^{(n-1)}\right)^{\prime}(f)\right) \\
& \quad=\operatorname{Res}\left(f^{(n)},\left(f^{(n-1)}\right)^{\prime}(f)\right) \operatorname{Res}\left(f^{(n)}, f^{\prime}\right) \\
& \quad=\left(f_{d}^{k d^{n-1} \frac{d^{n-1}-1}{d-1}} \operatorname{Res}\left(f^{(n-1)},\left(f^{(n-1)}\right)^{\prime}\right)\right)^{d} \operatorname{Res}\left(f^{(n)}, f^{\prime}\right) .
\end{aligned}
$$

Using Lemma 1(i), we derive

$$
\begin{aligned}
& \operatorname{Res}\left(f^{(n-1)},\left(f^{(n-1)}\right)^{\prime}\right) \\
& =(-1)^{\frac{d^{2}(d-1)}{2}} f_{d}^{\frac{d^{n-1}-1}{d-1}\left(-d^{n-1}+k \frac{d^{n-1}-1}{d-1}+2\right)} \operatorname{Disc}\left(f^{(n-1)}\right),
\end{aligned}
$$

while by Lemma 1(ii) we obtain

$$
\operatorname{Res}\left(f^{(n)}, f^{\prime}\right)=(-1)^{k d}\left((k+1) f_{k+1}\right)^{d^{n}} \prod_{i=1}^{k} f^{(n)}\left(\gamma_{i}\right) .
$$

Plugging (3) and (4) in (2) and using (1), we finish the proof.

For a polynomial $f=f_{d} X^{d}+\ldots+f_{1} X+f_{0} \in \mathbb{F}_{q}[X]$ defined as in Lemma 3 , it is convenient to introduce the following notation

$$
G_{n}\left(f_{d}, \ldots, f_{0}\right)=\prod_{i=1}^{k} f^{(n)}\left(\gamma_{i}\right), \quad n \geqslant 1,
$$

where $\gamma_{i}, i=1, \ldots, k$, are the roots of $f^{\prime}$, which is clearly a polynomial in $f_{d}, \ldots, f_{0}$. We need the following result, which has been proved in [8, Lemma 5.2]:

Lemma 4. For fixed integers $K \geqslant 1$ and $k_{1}, \ldots, k_{\mu}$ such that $1 \leqslant k_{1}<$ $\cdots<k_{\mu} \leqslant K$, the polynomial

$$
\prod_{j=1}^{\mu} G_{k_{j}}\left(f_{d}, \ldots, f_{0}\right)
$$


is a square polynomial in the variable $f_{0}$ up to a multiplicative constant only for $O\left(d^{2 K} q^{d-1}\right)$ choices of $f_{1}, \ldots, f_{d}$.

\section{Bounds of Some Character Sums}

We assume first that $d$ is even. In this situation, for an integer $n$, we consider the following character sum

$$
\begin{aligned}
& T_{1}(n)=\sum_{f_{0} \in \mathbb{F}_{q}} \ldots \sum_{f_{d} \in \mathbb{F}_{q}}\left|\sum_{\ell=1}^{n} \chi\left(G_{\ell}\left(f_{d}, \ldots, f_{0}\right) G_{\ell+1}\left(f_{d}, \ldots, f_{0}\right)\right)\right|^{2}, \\
& T_{2}(n)=\sum_{f_{0} \in \mathbb{F}_{q}} \ldots \sum_{f_{d} \in \mathbb{F}_{q}}\left|\sum_{\ell=1}^{n} \chi\left(f_{d}^{k \ell} G_{\ell}\left(f_{d}, \ldots, f_{0}\right)\right)\right|^{2},
\end{aligned}
$$

where $\chi$ is a quadratic character and $k$ is as in Lemma 3.

Lemma 5. Let $f=f_{d} X^{d}+\ldots+f_{1} X+f_{0} \in \mathbb{F}_{q}[X]$ be defined as in Lemma 3. For any integer $n \geqslant 1$, we have the following bound:

$$
T_{1}(n)=O\left(n^{2} d^{n} q^{d+1 / 2}+n^{2} d^{2 n} q^{d}+n q^{d+1}\right),
$$

and the same estimate holds for $T_{2}(n)$.

Proof. Squaring and changing the order of summation, we obtain

$$
\begin{array}{r}
T_{1}(n)=\sum_{\ell, m=1}^{n} \sum_{f_{d} \in \mathbb{F}_{q}} \ldots \sum_{f_{0} \in \mathbb{F}_{q}} \chi\left(G_{\ell}\left(f_{d}, \ldots, f_{0}\right) G_{\ell+1}\left(f_{d}, \ldots, f_{0}\right)\right. \\
\left.\cdot G_{m}\left(f_{d}, \ldots, f_{0}\right) G_{m+1}\left(f_{d}, \ldots, f_{0}\right)\right) .
\end{array}
$$

Fix $l, m, f_{1}, \ldots, f_{d}$ and define the following polynomial in $d_{0}$,

$$
G_{\ell, m}=G_{\ell}\left(f_{d}, \ldots, f_{0}\right) G_{\ell+1}\left(f_{d}, \ldots, f_{0}\right) G_{m}\left(f_{d}, \ldots, f_{0}\right) G_{m+1}\left(f_{d}, \ldots, f_{0}\right) .
$$

If $G_{\ell, m}$ is not a square polynomial in $f_{0}$ we use the Weil bound (see [9, Theorem 11.23]) and estimate the sum over $f_{0}$ as $O\left(d^{n} q^{1 / 2}\right)$. Otherwise, we use the trivial estimate and that gives $q$ for the sum over $f_{0}$. For $O\left(n^{2}\right)$ values of $\ell \neq m$ and $O\left(q^{d}\right)$ choices of $f_{1}, \ldots, f_{d}$, we apply the Weil bound, so the total contribution from all such terms is $O\left(n^{2} d^{n} q^{d+1 / 2}\right)$.

We know that $G_{m, l}$ is an square polynomial for $O\left(n^{2} d^{2 n} q^{d-1}\right)$ values of the fixed parameters $f_{1}, \ldots, f_{d}$ by Lemma 4 when $m \neq l$. Trivially, there are $n q^{d}$ possible choices for $f_{1}, \ldots, f_{d}$ with $l=m$. Then,

$$
T_{1}(n)=O\left(q \dot{n}^{2} d^{2 n} q^{d-1}+q \dot{n} q^{d}+d^{n} q^{1 / 2} \dot{n}^{2} q^{d}\right),
$$

and first part of the result now follows.

Following the same argument (with some natural simplifications due to a similar shape of the sum $\left.T_{2}(n)\right)$, we obtain the same estimate for $T_{2}(n)$. 


\section{Polynomial $A B C$-Theorem and Divisors of Iterated POLYNOMIALS}

Some of our results are also based on the Mason theorem [12] that gives a polynomial version of the $A B C$-conjecture, see also [17].

For a polynomial $f \in \mathbb{F}_{q}[X]$ we use $\operatorname{rad}(f)$ to denote the product of all monic irreducible divisors of $f$.

Lemma 6. Let $A, B, C$ be nonzero polynomials over $\mathbb{F}_{q}$ with $A+B+$ $C=0$ and $\operatorname{gcd}(A, B, C)=1$. If $\operatorname{deg} A \geqslant \operatorname{deg} \operatorname{rad}(A B C)$, then $A^{\prime}=0$.

Recall that we use $D_{n}(f)$ to denote the largest degree of irreducible factors of $f^{(n)}$. In order to apply Lemma 6 we need the following simple statement.

Lemma 7. Let $f \in \mathbb{F}_{q}[X]$ be a nonconstant polynomial, then

$$
D_{n}(f) \geqslant D_{n-1}(f)
$$

for $n \geqslant 2$.

Proof. Now assume that $D_{n-1}(f)=D$ for some positive integer $D$. Let $g \in \mathbb{F}_{q}[X]$ be an irreducible divisor of $f^{(n-1)}$ with $\operatorname{deg} g=D$. Then we obviously have $g(f) \mid f^{(n)}$. Now, if $g(f)$ has a root $\alpha \in \mathbb{F}_{q^{m}}$ then $g$ has a root $f(\alpha)$ in $\mathbb{F}_{q^{m}}$ too. Because $g$ is irreducible, we have $m \geqslant \operatorname{deg} g$. Thus $g(f)$ has an irreducible factor of degree at least $D$.

We denote by $\Delta_{n}(f)$ denote the largest degree of square-free divisors of $f^{(n)}$, that is, $\Delta_{n}(f)=\operatorname{deg} \operatorname{rad}\left(f^{(n)}\right)$.

Lemma 8. Let $f \in \mathbb{F}_{q}[X]$ be a nonconstant polynomial, then

$$
\Delta_{n}(f) \geqslant \Delta_{n-1}(f)
$$

for $n \geqslant 2$.

Proof. Assume that

$$
f^{(n-1)}=\prod_{i=1}^{s} g_{i}^{\alpha_{i}}
$$

where $g_{1}, \ldots, g_{s}$ are distinct irreducible divisors of $f^{(n-1)}$ of multiplicities $\alpha_{1}, \ldots, \alpha_{s}$, respectively,

$$
\Delta_{n-1}(f)=\sum_{i=1}^{s} \operatorname{deg} g_{i} .
$$

Then

$$
f^{(n)}=\prod_{i=1}^{s} g_{i}(f)^{\alpha_{i}} .
$$


As $g_{1}, \ldots, g_{s}$ are relatively prime, we see from Lemma 2 that the polynomials $g_{1}(f), \ldots, g_{s}(f)$ are relatively prime. Thus

$$
\Delta_{n}(f)=\sum_{i=1}^{s} \operatorname{deg} \operatorname{rad}\left(g_{i}(f)\right) .
$$

As in the proof of Lemma 7 we see that $\operatorname{deg} \operatorname{rad}\left(g_{i}(f)\right) \geqslant \operatorname{deg} g_{i}, i=$ $1, \ldots, s$, which concludes the proof.

\section{Growth of the Number of Irreducible Factors under ITERATIONS FOR SMALL $n$}

Let $f \in \mathbb{F}_{q}[X]$. We recall that $r_{n}(f)$ denotes the number of monic irreducible divisors of $f^{(n)}$. Using the remark after Lemma 2, we have that if $g_{1}, g_{2}$ are two different irreducible prime factors of $f^{(n)}$, then $g_{1}(f)$ and $g_{2}(f)$ are co-prime.

Clearly, this means that $r_{n}(f)$ is a non decreasing function and now, we show that $r_{n}(f)$ grows at least linearly for $n$ of order up to $\log q$.

Theorem 9. If $q$ is odd then for any fixed $\varepsilon>0$ for all but $o\left(q^{d+1}\right)$ polynomials $f \in \mathbb{F}_{q}[X]$ of degree $d$, we have

$$
r_{n}(f) \geqslant(0.5+o(1)) n,
$$

when $n \rightarrow \infty$ and $L \geqslant n$, where

$$
L=\left\lceil\left(\frac{1}{2 \log d}-\varepsilon\right) \log q\right\rceil .
$$

Proof. Clearly we can discard $q^{d}$ polynomials $f$ with $f(0)=0$.

We consider first the case when $d$ is even. In this case,

$$
\chi\left(G_{\ell}\left(f_{d}, \ldots, f_{0}\right)\right)=\chi\left(\operatorname{Disc}\left(f^{(\ell)}\right)\right)
$$

Let us apply Lemma 5 with $n \leqslant L$. Note that $d^{2 n}=O\left(q^{1-2 \varepsilon \log d}\right)$ and thus $T_{1}(n)=O\left(n q^{d+1}\right)$. Therefore the number of tuples $\left(f_{d}, \ldots, f_{0}\right) \in$ $\mathbb{F}_{q}^{d+1}$ with

$$
\left|\sum_{\ell=1}^{n} \chi\left(G_{\ell}\left(f_{d}, \ldots, f_{0}\right) G_{\ell+1}\left(f_{d}, \ldots, f_{0}\right)\right)\right| \geqslant n^{2 / 3}
$$

does not exceed $T_{1}(n) n^{-4 / 3}=O\left(q^{d+1} n^{-1 / 3}\right)=o\left(q^{d+1}\right)$ when $n \rightarrow \infty$.

So we discard $o\left(q^{d+1}\right)$ polynomials $f=f_{d} X^{d}+\ldots+f_{1} X+f_{0} \in \mathbb{F}_{q}[X]$, which correspond to such tuples $\left(f_{d}, \ldots, f_{0}\right)$.

We also discard the polynomials $f=f_{d} X^{d}+\ldots+f_{1} X+f_{0} \in \mathbb{F}_{q}[X]$ corresponding to tuples $\left(f_{d}, \ldots, f_{0}\right)$ for which

$$
G_{\ell}\left(f_{d}, \ldots, f_{0}\right) \cdot G_{\ell+1}\left(f_{d}, \ldots, f_{0}\right)=0
$$


for some $\ell=1, \ldots, n$. The 0 polynomial is an square polynomial, so using Lemma 4, we derive that there are $O\left(n d^{2 n} q^{d}\right)=o\left(q^{d+1}\right)$ such polynomials.

For the remaining polynomials, we have

$$
\chi\left(G_{\ell}\left(f_{d}, \ldots, f_{0}\right) G_{\ell+1}\left(f_{d}, \ldots, f_{0}\right)\right) \neq 0,
$$

and also

$$
\left|\sum_{\ell=1}^{n} \chi\left(G_{\ell}\left(f_{d}, \ldots, f_{0}\right) G_{\ell+1}\left(f_{d}, \ldots, f_{0}\right)\right)\right|<n^{2 / 3} .
$$

Thus, for these polynomials we have

$$
\chi\left(G_{\ell}\left(f_{d}, \ldots, f_{0}\right) G_{\ell+1}\left(f_{d}, \ldots, f_{0}\right)\right)=-1
$$

for $n / 2+O\left(n^{2 / 3}\right)$ values of $\ell=1, \ldots, n$. We now see from Lemma 3 that

$$
\chi\left(\operatorname{Disc}\left(f^{(\ell)}\right)\right) \neq \chi\left(\operatorname{Disc}\left(f^{(\ell+1)}\right)\right)
$$

for $n / 2+O\left(n^{2 / 3}\right)$ values of $\ell=1, \ldots, n$.

We use now the Stickelberger's theorem (see [18] or a recent reference [3]) which says that the number $r_{\ell}$ of distinct irreducible factors of $f^{(\ell)}$ satisfies $r_{\ell}(f) \equiv d^{\ell}(\bmod 2)$ if and only if Disc $\left(f^{(\ell)}\right)$ is a square in $\mathbb{F}_{q}$.

By (6), the fact that the degree is even and using the Stickelberger's theorem [18], $r_{\ell}(f)$ and $r_{\ell+1}(f)$ are of different parity for $n / 2+O\left(n^{2 / 3}\right)$ values of $\ell=1, \ldots, n$. Since clearly $r_{\ell}(f)$ is non decreasing, we have $r_{\ell+1}(f)>r_{\ell}(f)$ for such values of $\ell$. Thus

$$
r_{n}(f) \geqslant n / 2+O\left(n^{2 / 3}\right) .
$$

For odd $d$ we note that $r_{\ell}(f)$ and $r_{\ell+1}(f)$ are of different parity when $\chi\left(f_{d}^{k \ell} G_{\ell}\left(f_{d}, \ldots, f_{0}\right)\right)=-1$ and proceed exactly the same way using Lemma 5 .

\section{Lower Bound on the Degree of Irreducible Factors of ITERATES FOR LARGE $n$}

Recall that for a polynomial $f \in \mathbb{F}_{q}[X]$ we use $D_{n}(f)$ to denote the largest degree of irreducible factors of $f^{(n)}$.

We our now ready to prove our main result of this section.

Theorem 10. Let $f \in \mathbb{F}_{q}[X]$ be of degree $d$ with $\operatorname{gcd}(d, q)=1$ and such that $f \neq f_{d} X^{d}$. Then

$$
D_{n}(f)>\frac{\log \left(d^{n-1} / 2\right)}{\log q}
$$


Proof. We fix some integer $n$ and define $D$ as the largest integer satisfying

$$
2 q^{D} \leqslant d^{n-1}
$$

We prove the statement by contradiction, so we suppose that $D_{n}(f) \leqslant$ $D$. By Lemma 7 we have

$$
D_{n-1}(f) \leqslant D_{n}(f) .
$$

This means that the polynomial $f^{(n)} f^{(n-1)}$ can be factorized by irreducible polynomials of degree at most $D$.

Any root of $f^{(n)}$ or $f^{(n-1)}$ belongs to $\mathbb{F}_{q^{j}}$ with $j \leqslant D$. Then, the product $f^{(n)} f^{(n-1)}$ has at most

$$
\sum_{j=1}^{D} q^{j} \leqslant 2 q^{D}
$$

distinct roots.

Clearly, $f$ has a root $\alpha \neq 0$ in some extension field of $\mathbb{F}_{q}$, so $G \mid f$, where $G=X-\alpha$.

Furthermore, we can write

$$
f^{(n-1)}-G\left(f^{(n-1)}\right)-\alpha=0
$$

and apply Lemma 6 with $A=f^{(n-1)}, B=-G\left(f^{(n-1)}\right)$ and $C=-\alpha$. Using that $G\left(f^{(n-1)}\right) \mid f\left(f^{(n-1)}\right)=f^{(n)}$ we derive

$$
d^{n-1}<\operatorname{deg} \operatorname{rad}\left(G\left(f^{(n-1)}\right) f^{(n-1)}\right) \leqslant \operatorname{deg} \operatorname{rad}\left(f^{(n)} f^{(n-1)}\right) \leqslant 2 q^{D} .
$$

Hence we obtain $d^{n-1}<2 q^{D}$, which contradicts the choice of $D$.

\section{UNIFORM BOUND}

Note that Theorem 10 becomes nontrivial for $n$ of about the same level when Theorem 9 stops working. So they can be combined in the following result that provides some nontrivial information about the arithmetic structure of iterations that applies to all $n$ and $q$. Let, as before, $\Delta_{n}(f)$ denote the largest degree of square-free divisors of $f^{(n)}$.

Theorem 11. If $q$ is odd and $\operatorname{gcd}(d, q)=1$ then, for any fixed $\varepsilon>0$, for all but $o\left(q^{d+1}\right)$ polynomials $f \in \mathbb{F}_{q}[X]$ of degree $d$, for $n \geqslant 1$, we have

$$
\Delta_{n}(f) \gg n^{1-\varepsilon}
$$


Proof. First of all, we note that the proof of Theorem 9 and equation (5) show that for any fixed $\varepsilon>0$, for all but $o\left(q^{d+1}\right)$ polynomials $f \in \mathbb{F}_{q}[X]$ of degree $d, n \leqslant L$ with

$$
L=\left\lceil\left(\frac{1}{2 \log d}-\varepsilon\right) \log q\right\rceil,
$$

we have Disc $\left(f^{(n)}\right) \neq 0$ and thus $\Delta_{n}(f)=d^{n}$.

Therefore for every $n \leqslant q^{1 / 2}$, since by Lemma 8 , we know that $\Delta_{n}(f)$ is monotonic, by Theorem 9 for all but $o\left(q^{d+1}\right)$ polynomials $f \in \mathbb{F}_{q}[X]$ of degree $d$ we have

$$
\Delta_{n}(f) \geqslant \min \left\{d^{n}, d^{L}\right\} \gg n^{1-\varepsilon} .
$$

For $n>q^{1 / 2}$, by Theorem 10, for all but $O(q)=o\left(q^{d+1}\right)$ polynomials $f \in \mathbb{F}_{q}[X]$ of degree $d$ we have

$$
\Delta_{n}(f) \geqslant D_{n}(f) \gg \frac{1}{\log q} n \gg \frac{n}{\log n} \gg n^{1-\varepsilon} .
$$

Combining (8) and (9), we conclude the proof.

\section{Comments and Open Questions}

We note that an analogue of Theorems 9 and 11 can be obtained for almost all monic polynomials as well. Probably the most interesting question is to extend the bound of Theorem 9 to any $n$ (beyond of the current threshold $n=O(\log q))$.

In [11] the critical orbit of quadratic polynomials $f$ is defined as the set $\left\{f^{(n)}(\gamma) \mid n \geqslant 2\right\} \cup\{-f(\gamma)\}$, where $\gamma$ is the root of the derivative. This coincides with the following set

$$
\left\{G_{n}\left(f_{0}, f_{1}, f_{2}\right) \mid n \geqslant 2\right\} \cup\left\{\frac{f_{1}^{2}}{2 f_{0}}-f_{2}\right\} .
$$

It is certainly interesting to investigate various properties of the sequence $u_{n}=G_{n}\left(f_{0}, \ldots, f_{d}\right)$ for $f_{0}, \ldots, f_{d} \in \mathbb{F}_{q}$ fixed.

At this moment, most of the known results concern only quadratic polynomials are known. For example, the sequence $u_{n}$ becomes eventually periodic when $d=2$. If $f^{\prime}$ is a irreducible polynomial of degree $k$, then $G_{n}\left(f_{0}, \ldots, f_{d}\right)=\operatorname{Norm}_{\mathbb{F}_{q^{k}} / \mathbb{F}_{q}}\left(f^{(n)}(\gamma)\right)$ is the norm of $f^{(n)}(\gamma)$ in $\mathbb{F}_{q}$. Apart from these two cases, very little is known about the sequence $u_{n}$ for general polynomials $f$.

The sparsity, or number of monomials, is another important characteristic of polynomials and it is certainly interesting to obtain lower 
bounds on the number of monomials of the iterations $f^{(n)}$. For iterations of polynomials and even rational functions over a field of characteristic zero such bounds can be derived from the results of [5].

Finally, we note that similar questions can also be asked for iterations of rational functions, which is yet another challenging direction of research.

\section{ACKNOWLEDGEMENT}

The author would like to thank the referee for his very careful reading and many suggestions which have improve the quality of the exposition. D. G.-P. would like to thank Macquarie University for its hospitality and support during his visit in 2012, when this work was initiated. During the preparation of this paper, D. G.-P. was partially supported by the Spanish Government Projects MTM2011-24678 and TIN201127479-C04-04, A. O. by the Swiss National Foundation Grant PA00P2139679, and I. S. by the Australian Research Grant DP1092835.

\section{REFERENCES}

[1] N. Ali, 'Stabilité des polynômes', Acta Arith., 119 (2005), 53-63.

[2] M. Ayad and D. L. McQuillan, 'Irreducibility of the iterates of a quadratic polynomial over a field', Acta Arith., 93 (2000), 87-97; Corrigendum: Acta Arith., 99 (2001), 97.

[3] K. Dalen, 'On a theorem of Stickelberger', Math. Scand., 3 (1955), 124-126.

[4] X. Faber and A. Granville, 'Prime factors of dynamical sequences', J. Reine Angew. Math., 661 (2011), 189-214.

[5] C. Fuchs and U. Zannier, 'Composite rational functions expressible with few terms', J. Eur. Math. Soc., 14 (2012), 175-208.

[6] J. von zur Gathen and J. Gerhard. Modern computer algebra, Cambridge University Press, 1999.

[7] D. Gomez-Perez and A. P. Nicolás, 'An estimate on the number of stable quadratic polynomials', Finite Fields and Appl., 16 (2010), 329-333.

[8] D. Gomez-Perez, A. P. Nicolás, A. Ostafe and D. Sadornil, 'Stable polynomials over finite fields', Preprint, 2011.

[9] H. Iwaniec and E. Kowalski, Analytic number theory, Amer. Math. Soc., Providence, RI, 2004.

[10] R. Jones, 'The density of prime divisors in the arithmetic dynamics of quadratic polynomials', J. Lond. Math. Soc., 78 (2008), 523-544.

[11] R. Jones and N. Boston, 'Settled polynomials over finite fields,' Proc. Amer. Math. Soc., 140 (2012), 1849-1863.

[12] R. C. Mason, Diophantine Equations over Functions Fields, Cambridge, Cambridge Univ.Press, 1984.

[13] J. H. McKay and S. S.-S. Wang, 'A chain rule for the resultant of two polynomials', Archiv Math., 53 (1989), 347-351.

[14] R. W. K. Odoni, 'The Galois theory of iterates and composites of polynomials,' Proc. London Math. Soc., 51 (1985), 385-414. 
12 DOMINGO GÓMEZ-PÉREZ, ALINA OSTAFE, AND IGOR E. SHPARLINSKI

[15] A. Ostafe and I. E. Shparlinski, 'On the length of critical orbits of stable quadratic polynomials', Proc. Amer. Math. Soc., 138 (2010), 2653-2656.

[16] I. E. Shparlinski, 'The number of different prime divisors of recurrent sequences', Mat. Zametki, 42 (1987), 494-507 (in Russian).

[17] N. Snyder, 'An alternate proof of Mason's theorem', Elemente Math., 55 (2000), 93-94.

[18] L. Stickelberger, 'Über eine neue Eigenschaft der Diskriminanten algebraischer Zahlkörper', Verh. 1 Internat. Math. Kongresses, 1897, Leipzig, 1898, 182-193.

[19] R. G. Swan, 'Factorization of polynomials over finite fields', Pacific J. Math., 12 (1962), 1099-1106.

Department of Mathematics, University of Cantabria, Santander 39005, SPAIN

E-mail address: domingo.gomez@unican.es

Department of Computing, Macquarie University, Sydney NSW 2109, Australia

E-mail address: alina.ostafe@mq.edu.au

Department of Computing, Macquarie University, Sydney NSW 2109, Australia

E-mail address: igor.shparlinski@mq.edu.au 